\title{
Discriminatory Attitudes of Pharmacy Students and Pharmacists against People Living with HIVIAIDS
}

\author{
Chukwuemeka Michael Ubaka*, Maxwell Ogochukwu Adibe and Chinwe \\ Victoria Ukwe \\ Department of Clinical Pharmacy and Pharmacy Management, University of Nigeria, Nsukka, Nigeria.
}

*For correspondence: Email: pharmubk@yahoo.com, chukwuemeka.ubaka@unn.edu.ng; Tel: +234 8038246913

\begin{abstract}
Purpose: To evaluate the level and predictors of discriminatory attitudes of pharmacy students and pharmacists towards people living with HIVIAIDS (PLWHA).

Methods: A cross-sectional survey of pharmacy students and pharmacists $(n=523)$ to assess discriminatory attitudes towards PLWHA was conducted using a self completed questionnaire. Correlation and regression analyses were conducted.

Results: Pharmacists were more knowledgeable and had less negative perceptions than students towards PLWHA. Level of professional training $(p<0.0001)$, knowledge status $(p<0.0001)$ and five negative perception items, namely, (a) people who got HIV through sex deserve it $(p=0.003)$, (b) PLWHA would make their colleagues apprehensive $(p<0.0001)$, (c) PLWHA have poor hygiene ( $r=$ $0.082, p=0.032)$, (d) PLWHA should feel ashamed of themselves $(p<0.0001)$, and $(e)$ people who behave promiscuously should be blamed for AIDS $(p=0.031)$, were all significantly associated with higher discrimination. However, being a student and having negative perceptions such as "PLWHA should feel ashamed of themselves and "PLWHA would make their colleagues apprehensive" were independent predictors of discrimination.

Conclusions: Discriminatory attitudes against PLWHA among pharmacy students and pharmacists who participated in this study were high and level of training and their perceptions were contributory.
\end{abstract}

Keywords: Discrimination, HIVIAIDS, Pharmacists, Perception, Professionalism, Stigmatization.

Tropical Journal of Pharmaceutical Research is indexed by Science Citation Index (SciSearch), Scopus, International Pharmaceutical Abstract, Chemical Abstracts, Embase, Index Copernicus, EBSCO, African Index Medicus, JournalSeek, Journal Citation Reports/Science Edition, Directory of Open Access Journals (DOAJ), African Journal Online, Bioline International, Open-J-Gate and Pharmacy Abstracts

\section{INTRODUCTION}

Recent statistics show that the incidence of new HIV infections worldwide tends to be on the decline but the stigmatization associated with the disease and those affected continues to be on the increase [1]. More worrisome is the high prevalence of stigmatization and eventual discrimination of people living with HIVIAIDS (PLWHA) among health professionals who have direct contact with patients' body fluids [2-6]. Studies showing varying levels of high discriminatory attitudes among doctors [2,3], nurses [4,5], midwives [5] and physiotherapists [6] have been reported in literature. These attitudes have consistently been found to pose as impediments to successful testing and treatment services offered this group of patients $[7,8]$.

Though significant progress has been made in measuring stigma associated with HIV, studies of the prevalence of stigma among health professionals (let alone at different levels of education) are still rare. Few studies assessing pharmacists and students' discriminatory 
attitudes towards PLWHA have been documented. Chisholm and Ricci developed and validated an instrument assessing first year pharmacy students' attitude towards PLWHA in a US school of pharmacy and found discriminatory attitudes to be prevalent amongst these students [9]. Ahmed et al explored levels of pharmacy students' attitude and risk perception of HIVIAIDS between Malay and Chinese students, and found discriminating attitudes and perceptions to be common [10]. In Ghana, a study conducted to assess the perceptions of Ghanaian pharmacists towards PLWHA found only $17 \%$ of pharmacists having acceptable attitudes towards them [11]. Letamo, in a 2005 Nigerian survey, assessed health care professionals' discriminatory attitudes towards PLWHA but did not include pharmacists [5].

With the apparent inadequacy of studies assessing discriminatory attitudes among pharmacists and especially in a highly endemic country like Nigeria, it would be difficult to truly actualize the mandate of equitable and assessable health care delivery to the ailing population. Our study therefore sought to provide answers to three study questions: (1) does discrimination of PLWHA exist among pharmacy students and pharmacists? (2) Are negative perceptions towards PLWHA and poor knowledge of HIVIAIDS among pharmacists and students associated with discriminatory attitudes, if any, towards them? (3) Does the level of training and experience influence these discriminatory attitudes if they exist?

\section{METHODS}

A cross sectional survey testing discriminatory attitudes towards PLWHA was conducted among pharmacy students at the University of Nigeria, Nsukka and registered pharmacists at the Federal and State University Teaching Hospitals in Enugu state in September 2011. The Human and Research Ethics Board of the University of Nigeria reviewed and approved this study.

As at 2011, there were about 130 registered pharmacists at both the University of Nigeria Teaching Hospital (Federal) and Enugu State University of Technology Hospital (state) with majority of them being graduates of the aforementioned university.

A 34 item survey instrument was generated from previous surveys involving pharmacy students and a general population which assessed discriminatory attitudes [10,12]. Fifteen item questions assessed respondents' knowledge of HIVIAIDS, HIV transmission and treatment. Fourteen item statements assessed discriminatory attitudes towards PLWHA while 5 item statements assessed perceptions towards PLWHA. Items on discriminatory attitudes were used to generate the discriminatory attitude score which represents each respondent's level of discrimination towards PLWHA, with knowledge and perception items acting as associating factors. Knowledge items were given binary response scales (yes/no) while discriminatory attitude and perception items were scored on 4-point Likert scale (agree/disagree). Reliability estimate of the questionnaires domain were reported using Cronbach's alpha.

Sampling of both students and pharmacists was by convenience. Students selected include those in their first professional year in pharmacy (preclinical) with no formal lecture on HIVIAIDS and those in the penultimate and final year classes (clinical) having received lectures on various topics on HIVIAIDS. Only registered pharmacists who have daily contact with PLWHA were selected for this study. Study participants were approached during a compulsory lecture (for students) and after dispensing of drugs to patients (for pharmacists). Oral consent of participation obtained before issuing the survey instrument. Sufficient time (maximum of 30 minutes) was given for the completion of the survey instrument.

\section{Data analysis}

Data from the retrieved questionnaires were subjected to differential and inferential analysis using SPSS software, version 16.0 (SPSS Inc, Chicago, USA). Age category was defined as "young" for students less than 25 years and pharmacists less than 40 years. Knowledge status was determined by the sum of correct responses for each respondent. Discriminatory attitudes were obtained by coding the discriminatory response with a higher value for each item and a composite score (Discriminatory Attitude Score, DAS) for each respondent derived by the mean of all discriminatory responses (thus the higher the score the more the discrimination). Descriptive statistics (frequency) were conducted for knowledge, discriminatory attitude and perception items categorized according to demographic characteristics (age, gender and professional training). In order to show relationship between discriminatory attitudes (DAS, a continuous variable) and variables such as demographic variables, knowledge status and negative perceptions, a correlation analysis (Pearson) was conducted to examine their association. Both simple and multiple linear regression analysis were conducted to further explore the variables 
that independently predicted discrimination towards PLWHA.

\section{RESULTS}

\section{Demographic characteristics and partici- pation rates}

Five hundred and forty four respondents participated in the study (an overall participation rate of $78.3 \%$ ). Data for 21 respondents were discarded for incompleteness in demographic information, leaving only 523 valid questionnaires. Of these valid respondents, 180 (34.4\%) were pre-clinical students, 272 (52.0\%) were clinical students and $71(13.6 \%)$ practicing pharmacists.

There were more males (55.6\%) among the respondents with the gender (female vs male) distribution across participating groups seen as; pre-clinical students (42.2 vs. $57.8 \%$ ), clinical students (45.2 vs. $54.8 \%$ ) and pharmacists (47.9 vs. $52.1 \%)$. There were also much younger participants (i.e. defined as $<25$ years for students and $<40$ years for pharmacists) than their older counterparts in this study (82.8 vs. $17.2 \%)$.

\section{Knowledge of HIVIAIDS among respondents}

Of the 15 knowledge items, there was poor knowledge (i.e., < $50 \%$ correct response) in three items. A high percentage of all the participant groups were knowledgeable of the transmission (routes) of HIV, giving at least $70 \%$ correct answers to items addressing HIV transmission. HIVIAIDS knowledge increased as level of professional training increased, with pharmacists displaying the best knowledge of the disease; this was also applicable to all items (Table 1). There was however, poor knowledge (< $50 \%$ correctness) among pre-clinical and clinical students in item questions on the use of antiretroviral agents.

\section{Negative perceptions against PLWHA}

Eighty one percent (425 of 523) of the respondents in this study possessed negative perceptions about PLWHA in one or more perception items. Majority of them believed promiscuity is the blame for HIV (56.7\%) and that PLWHA would get their non-infected colleagues apprehensive (51.2\%). Also noted was the decrease in these negative perceptions from pre-clinical students to pharmacists. A higher percentage of older pharmacists thought that PLWHA should feel ashamed of themselves (Table 2).

\section{Discriminatory attitudes towards PLWHA}

Overall, only $19.6 \%$ (103 of 523) of respondents had accepting attitudes for all the items tested. Of the fourteen items testing discriminating attitudes towards PLWHA, students and pharmacists showed discriminatory patterns (in more than $20 \%$ of the cases) in 5 items. However, pharmacists showed lower (31\% having accepting attitude in all items) discriminatory attitudes towards PLWHA. Male pre-clinical students showed significantly more discriminatory attitudes towards PLWHA than the females in 4 items. Other significant associations between demographics data and discriminatory attitudes are shown in Table 3.

\section{Factors influencing discriminatory attitudes against PLWHA}

When knowledge status and 3 negative perception items were adjusted against discriminatory attitudes towards PLWHA, it was seen that students/pharmacists with poor knowledge of HIVIAIDS were more likely to exhibit discriminatory attitudes in 6 attitude items (items 4, 7, 8, 9, 12 and 14). Respondents who perceived that people who got HIV through sex deserved it were more likely to display discriminatory attitudes in 3 items (items 1, 7 and 12). For those respondents who felt PLWHA should be ashamed of themselves, they were more likely to be discriminatory in 5 attitude items (items 3, 7, 12, 13 and 14). Lastly, students/pharmacists who perceived that PLWHA would make their colleagues apprehensive were more likely to be discriminatory in 5 attitude items (items $1,4,7$, 12 and 14) (Table 4).

Univariate analysis showed that only level of professional training $(r=-0.262, p<0.0001)$, knowledge status $(r=-0.183 ; p<0.0001)$ and 5 negative perception items, i.e., people who got HIV through sex deserve it $(r=0.122 ; p=0.003)$, PLWHA would make their colleagues apprehensive $(r=0.171 ; p<0.0001)$, PLWHA have a poor hygiene $(r=0.082, p=0.032)$, PLWHA should feel ashamed of themselves $(r=$ $0.177 ; p<0.0001)$, people who behave promiscuously should be blamed for AIDS ( $r=$ $0.083 ; p=0.031$ ) were significantly correlated with the DAS. However, stepwise multiple linear regression analysis produced three predictive models. 
Table 1: HIVIAIDS knowledge among pharmacy students and pharmacists by respondents' characteristics

\begin{tabular}{|c|c|c|c|c|c|c|c|c|c|c|c|c|c|}
\hline \multirow[t]{3}{*}{$\%$ Yes or no responses } & \multicolumn{4}{|c|}{ Pre-Clinical students } & \multicolumn{4}{|c|}{ Clinical students } & \multicolumn{4}{|c|}{ Pharmacists } & \multirow{3}{*}{$\begin{array}{c}\text { Overal } \\
I \%\end{array}$} \\
\hline & \multicolumn{2}{|c|}{$\begin{array}{l}\text { Gender } \\
(\%)\end{array}$} & \multicolumn{2}{|c|}{ Age (\%) } & \multicolumn{2}{|c|}{$\begin{array}{l}\text { Gender } \\
(\%)\end{array}$} & \multicolumn{2}{|c|}{ Age (\%) } & \multicolumn{2}{|c|}{$\begin{array}{l}\text { Gender } \\
(\%)\end{array}$} & \multicolumn{2}{|c|}{ Age (\%) } & \\
\hline & $\boldsymbol{F}$ & $M$ & $\leq 25$ & $>25$ & $F$ & $M$ & $\leq 25$ & $>25$ & $F$ & $M$ & $\leq 40$ & $>40$ & \\
\hline $\begin{array}{l}\text { 1. Can HIVIAIDS be transmitted } \\
\text { from infected mother to child?(Yes) }\end{array}$ & 86.8 & $\begin{array}{l}84 . \\
6\end{array}$ & $\begin{array}{r}85 . \\
2\end{array}$ & $\begin{array}{l}90 . \\
0\end{array}$ & $\begin{array}{r}91 . \\
9\end{array}$ & $\begin{array}{l}98 . \\
0\end{array}$ & $\begin{array}{r}95 . \\
1\end{array}$ & $\begin{array}{l}95 . \\
8\end{array}$ & $\begin{array}{r}97 . \\
1\end{array}$ & 100 & $\begin{array}{r}96 . \\
9\end{array}$ & 100 & 92.3 \\
\hline $\begin{array}{l}\text { 2. Can HIV be transmitted via air } \\
\text { and contact with water?(No) }\end{array}$ & 97.4 & $\begin{array}{l}97 . \\
1\end{array}$ & $\begin{array}{r}97 . \\
0\end{array}$ & 100 & $\begin{array}{r}97 . \\
6\end{array}$ & $\begin{array}{l}98 . \\
0\end{array}$ & $\begin{array}{r}97 . \\
0\end{array}$ & 100 & 100 & 100 & 100 & 100 & 98.0 \\
\hline $\begin{array}{l}\text { 3. Can HIV be transferred through } \\
\text { social contacts like sharing clothes, } \\
\text { Cups/plates/spoon/glass/bathrooms } \\
\text {, shaking hands and kissing?(No) }\end{array}$ & 82.9 & $\begin{array}{l}85 . \\
9\end{array}$ & $\begin{array}{r}84 . \\
0\end{array}$ & $\begin{array}{l}90 . \\
9\end{array}$ & $\begin{array}{r}82 . \\
9\end{array}$ & $\begin{array}{l}83 . \\
7\end{array}$ & $\begin{array}{r}82 . \\
8\end{array}$ & $\begin{array}{l}84 . \\
5\end{array}$ & $\begin{array}{r}97 . \\
1\end{array}$ & $\begin{array}{l}94 . \\
3\end{array}$ & $\begin{array}{r}95 . \\
2\end{array}$ & 100 & 85.6 \\
\hline $\begin{array}{l}\text { 4. Are unprotected sex, unscreened } \\
\text { blood, occupational exposures and } \\
\text { intravenous drug use major routes } \\
\text { of transmission?(Yes) }\end{array}$ & 97.4 & $\begin{array}{l}97 . \\
1\end{array}$ & $\begin{array}{r}97 . \\
6\end{array}$ & $\begin{array}{l}90 . \\
9\end{array}$ & $\begin{array}{r}98 . \\
4\end{array}$ & $\begin{array}{l}99 . \\
3\end{array}$ & $\begin{array}{r}99 . \\
0\end{array}$ & $\begin{array}{l}98 . \\
6\end{array}$ & 100 & 100 & 100 & 100 & 98.3 \\
\hline $\begin{array}{l}\text { 5. Do you think HIV can be } \\
\text { completely cured with available } \\
\text { antiretroviral therapy?(No) }\end{array}$ & 67.1 & $\begin{array}{l}77 . \\
9\end{array}$ & $\begin{array}{r}72 . \\
8\end{array}$ & $\begin{array}{l}81 . \\
8\end{array}$ & $\begin{array}{r}82 . \\
1\end{array}$ & $\begin{array}{l}85 \\
6\end{array}$ & $\begin{array}{r}82 . \\
3\end{array}$ & $\begin{array}{l}90 . \\
1\end{array}$ & $\begin{array}{r}85 . \\
7\end{array}$ & $\begin{array}{l}97 . \\
2\end{array}$ & $\begin{array}{r}89 . \\
1\end{array}$ & 100 & 81.6 \\
\hline $\begin{array}{l}\text { 6. Non Nucleosides/Nucleosides } \\
\text { Reverse Transcriptase and } \\
\text { Protease inhibitors are the most } \\
\text { widely available classes of HIV } \\
\text { medications(Yes) }\end{array}$ & 21.1 & $\begin{array}{l}35 . \\
6\end{array}$ & $\begin{array}{r}29 . \\
6\end{array}$ & $\begin{array}{l}27 . \\
3\end{array}$ & $\begin{array}{r}49 . \\
6\end{array}$ & $\begin{array}{l}62 . \\
7\end{array}$ & $\begin{array}{r}53 . \\
2\end{array}$ & $\begin{array}{l}67 . \\
6\end{array}$ & $\begin{array}{r}97 . \\
1\end{array}$ & $\begin{array}{l}91 . \\
9\end{array}$ & $\begin{array}{r}95 . \\
3\end{array}$ & $\begin{array}{l}90 . \\
0\end{array}$ & 52.7 \\
\hline $\begin{array}{l}\text { 7. Can antiviral agents like e.g. } \\
\text { (Acyclovir, Ribavirine, Amantadine) } \\
\text { be used to treat HIVIAIDS?(No) }\end{array}$ & 21.1 & $\begin{array}{l}33 . \\
7\end{array}$ & $\begin{array}{r}29 . \\
0\end{array}$ & $\begin{array}{l}18 . \\
2\end{array}$ & $\begin{array}{r}41 . \\
5\end{array}$ & $\begin{array}{l}42 . \\
5\end{array}$ & $\begin{array}{r}42 . \\
4\end{array}$ & $\begin{array}{l}40 . \\
8\end{array}$ & $\begin{array}{r}57 . \\
6\end{array}$ & $\begin{array}{l}80 . \\
0\end{array}$ & $\begin{array}{r}63 . \\
3\end{array}$ & $\begin{array}{l}90 . \\
0\end{array}$ & 41.0 \\
\hline $\begin{array}{l}\text { 8. Can a HIVIAIDS patient donate } \\
\text { blood? (No) }\end{array}$ & 92.1 & $\begin{array}{l}87 . \\
5\end{array}$ & $\begin{array}{r}89 . \\
9\end{array}$ & $\begin{array}{l}81 . \\
8\end{array}$ & $\begin{array}{r}91 . \\
1\end{array}$ & $\begin{array}{l}92 . \\
2\end{array}$ & $\begin{array}{r}91 . \\
6\end{array}$ & $\begin{array}{l}91 . \\
5\end{array}$ & 100 & $\begin{array}{l}97 . \\
1\end{array}$ & $\begin{array}{r}98 . \\
4\end{array}$ & 100 & 91.7 \\
\hline $\begin{array}{l}\text { 9. Are antiretrovirals recommended } \\
\text { for post exposure prophylaxis of } \\
\text { HIV?(Yes) }\end{array}$ & 22.4 & $\begin{array}{l}31 . \\
1\end{array}$ & $\begin{array}{r}26 . \\
2\end{array}$ & $\begin{array}{l}45 . \\
5\end{array}$ & $\begin{array}{r}56 . \\
9\end{array}$ & $\begin{array}{l}54 . \\
9\end{array}$ & $\begin{array}{r}55 . \\
2\end{array}$ & $\begin{array}{l}59 . \\
2\end{array}$ & $\begin{array}{r}87 . \\
9\end{array}$ & $\begin{array}{l}88 . \\
9\end{array}$ & 87. & 100 & 49.8 \\
\hline $\begin{array}{l}\text { 10. Are HIV and AIDS similar } \\
\text { things?(No) }\end{array}$ & 51.3 & $\begin{array}{l}51 . \\
9\end{array}$ & $\begin{array}{r}52 . \\
7\end{array}$ & $\begin{array}{l}36 . \\
4\end{array}$ & $\begin{array}{r}52 . \\
0\end{array}$ & $\begin{array}{l}64 . \\
7\end{array}$ & $\begin{array}{r}61 . \\
1\end{array}$ & $\begin{array}{l}53 . \\
5\end{array}$ & $\begin{array}{r}42 . \\
9\end{array}$ & $\begin{array}{l}75 . \\
0\end{array}$ & $\begin{array}{r}60 . \\
9\end{array}$ & $\begin{array}{l}60 . \\
0\end{array}$ & 56.9 \\
\hline $\begin{array}{l}\text { 11. Does avoiding mosquito bites } \\
\text { prevent HIV?(No) }\end{array}$ & 86.8 & $\begin{array}{l}83 . \\
7\end{array}$ & $\begin{array}{r}86 . \\
4\end{array}$ & $\begin{array}{l}63 . \\
6\end{array}$ & $\begin{array}{r}90 . \\
2\end{array}$ & $\begin{array}{l}93 . \\
5\end{array}$ & $\begin{array}{r}92 . \\
6\end{array}$ & $\begin{array}{l}90 . \\
1\end{array}$ & 100 & $\begin{array}{l}97 . \\
2\end{array}$ & $\begin{array}{r}98 . \\
4\end{array}$ & 100 & 90.7 \\
\hline $\begin{array}{l}\text { 12. Are bone infections, typhoid } \\
\text { fever and heart infections the major } \\
\text { infections in HIV patients that cause } \\
\text { death?(No) }\end{array}$ & 43.4 & $\begin{array}{l}46 . \\
2\end{array}$ & $\begin{array}{r}45 . \\
6\end{array}$ & $\begin{array}{l}36 . \\
4\end{array}$ & $\begin{array}{r}56 . \\
1\end{array}$ & $\begin{array}{l}57 . \\
5\end{array}$ & $\begin{array}{r}56 . \\
2\end{array}$ & $\begin{array}{l}56 . \\
3\end{array}$ & $\begin{array}{r}82 . \\
4\end{array}$ & $\begin{array}{l}72 . \\
2\end{array}$ & $\begin{array}{r}75 . \\
8\end{array}$ & $\begin{array}{l}90 . \\
0\end{array}$ & 55.5 \\
\hline $\begin{array}{l}\text { 13. Can food sharing with HIV } \\
\text { infected individuals transmit } \\
\text { HIV?(No) }\end{array}$ & 96.0 & $\begin{array}{l}94 . \\
2\end{array}$ & $\begin{array}{r}95 . \\
2\end{array}$ & $\begin{array}{l}90 . \\
9\end{array}$ & $\begin{array}{r}94 . \\
3\end{array}$ & $\begin{array}{l}93 . \\
5\end{array}$ & $\begin{array}{r}93 . \\
1\end{array}$ & $\begin{array}{l}95 . \\
8\end{array}$ & $\begin{array}{r}97 . \\
0\end{array}$ & $\begin{array}{l}97 . \\
2\end{array}$ & $\begin{array}{r}96 . \\
8\end{array}$ & 100 & 94.8 \\
\hline $\begin{array}{l}\text { 14. Can HIV infection develop into } \\
\text { AIDS within a year?(No) }\end{array}$ & 19.7 & $\begin{array}{l}34 . \\
6\end{array}$ & $\begin{array}{r}27 . \\
8\end{array}$ & $\begin{array}{l}36 . \\
4\end{array}$ & $\begin{array}{r}35 . \\
0\end{array}$ & $\begin{array}{l}41 . \\
2\end{array}$ & $\begin{array}{r}37 . \\
4\end{array}$ & $\begin{array}{l}42 . \\
3\end{array}$ & $\begin{array}{r}26 . \\
5\end{array}$ & $\begin{array}{l}45 . \\
5\end{array}$ & $\begin{array}{r}35 . \\
0\end{array}$ & $\begin{array}{l}40 . \\
0\end{array}$ & 34.9 \\
\hline $\begin{array}{l}\text { 15. Can contact with faeces, urine } \\
\text { saliva cause HIV?(No) }\end{array}$ & 67.1 & $\begin{array}{l}63 . \\
5\end{array}$ & $\begin{array}{r}65 . \\
1\end{array}$ & $\begin{array}{l}63 . \\
6\end{array}$ & $\begin{array}{r}75 . \\
2\end{array}$ & $\begin{array}{l}64 . \\
7\end{array}$ & $\begin{array}{r}68 . \\
8\end{array}$ & $\begin{array}{l}72 . \\
9\end{array}$ & $\begin{array}{r}79 . \\
4\end{array}$ & $\begin{array}{l}91 . \\
2\end{array}$ & $\begin{array}{r}85 . \\
2\end{array}$ & $\begin{array}{l}90 . \\
0\end{array}$ & 70.4 \\
\hline
\end{tabular}

Note: Responses in parenthesis represent correct answers

Table 2: Negative perceptions towards PLWHA among pharmacy students and pharmacists

\begin{tabular}{|c|c|c|c|c|c|c|c|c|c|c|c|c|c|}
\hline \multirow{3}{*}{$\begin{array}{l}\% \text { Agreeing or disagreeing with the } \\
\text { statements (perception) }\end{array}$} & \multicolumn{4}{|c|}{ Pre-Clinical students } & \multicolumn{4}{|c|}{ Clinical students } & \multicolumn{4}{|c|}{ Pharmacists } & \multirow{3}{*}{$\begin{array}{c}\text { Overall } \\
\quad \%\end{array}$} \\
\hline & \multicolumn{2}{|c|}{$\begin{array}{l}\text { Gender } \\
(\%)\end{array}$} & \multicolumn{2}{|c|}{ Age (\%) } & \multicolumn{2}{|c|}{$\begin{array}{l}\text { Gender } \\
(\%)\end{array}$} & \multicolumn{2}{|c|}{ Age (\%) } & \multicolumn{2}{|c|}{$\begin{array}{l}\text { Gender } \\
(\%)\end{array}$} & \multicolumn{2}{|c|}{ Age (\%) } & \\
\hline & $F$ & $M$ & $\leq 25$ & $>25$ & $F$ & $M$ & $\leq 25$ & $>25$ & $F$ & $M$ & $\leq 40$ & $>40$ & \\
\hline $\begin{array}{l}\text { 1. People who got HIVIAIDS through } \\
\text { sex or drug use got what they } \\
\text { deserved (agree) }\end{array}$ & 29.6 & 21.0 & 24.7 & 22.2 & 18.7 & 21.9 & 22.3 & 14.3 & 9.1 & 10.8 & 9.4 & 11.1 & 20.0 \\
\hline $\begin{array}{l}\text { 2. PLWHA should feel ashamed of } \\
\text { themselves (agree) }\end{array}$ & 11.3 & 15.8 & 14.1 & 11.1 & 8.1 & 6.6 & 7.4 & 8.6 & 0.0 & 2.7 & 1.6 & 10.0 & 8.9 \\
\hline $\begin{array}{l}\text { 3. People who behave promiscuously } \\
\text { should be blamed for AIDS (agree) }\end{array}$ & 59.2 & 60.0 & 58.6 & 77.8 & 56.9 & 60.3 & 61.4 & 50.0 & 51.5 & 37.8 & 45.3 & 22.2 & 56.7 \\
\hline $\begin{array}{l}\text { 4. PLWHA have a poor personal } \\
\text { hygiene (agree) }\end{array}$ & 12.7 & 15.8 & 13.5 & 33.3 & 11.5 & 12.6 & 12.4 & 11.4 & 8.8 & 5.6 & 7.9 & 0.0 & 11.9 \\
\hline $\begin{array}{l}\text { 5. PLWHA would make other } \\
\text { colleagues apprehensive (agree) }\end{array}$ & 54.3 & 51.5 & 53.1 & 44.4 & 58.7 & 49.7 & 53.5 & 53.6 & 43.8 & 38.2 & 44.1 & 20.0 & 51.2 \\
\hline
\end{tabular}


Table 3: Discriminatory attitudes towards PLWHA among pharmacy students and pharmacists

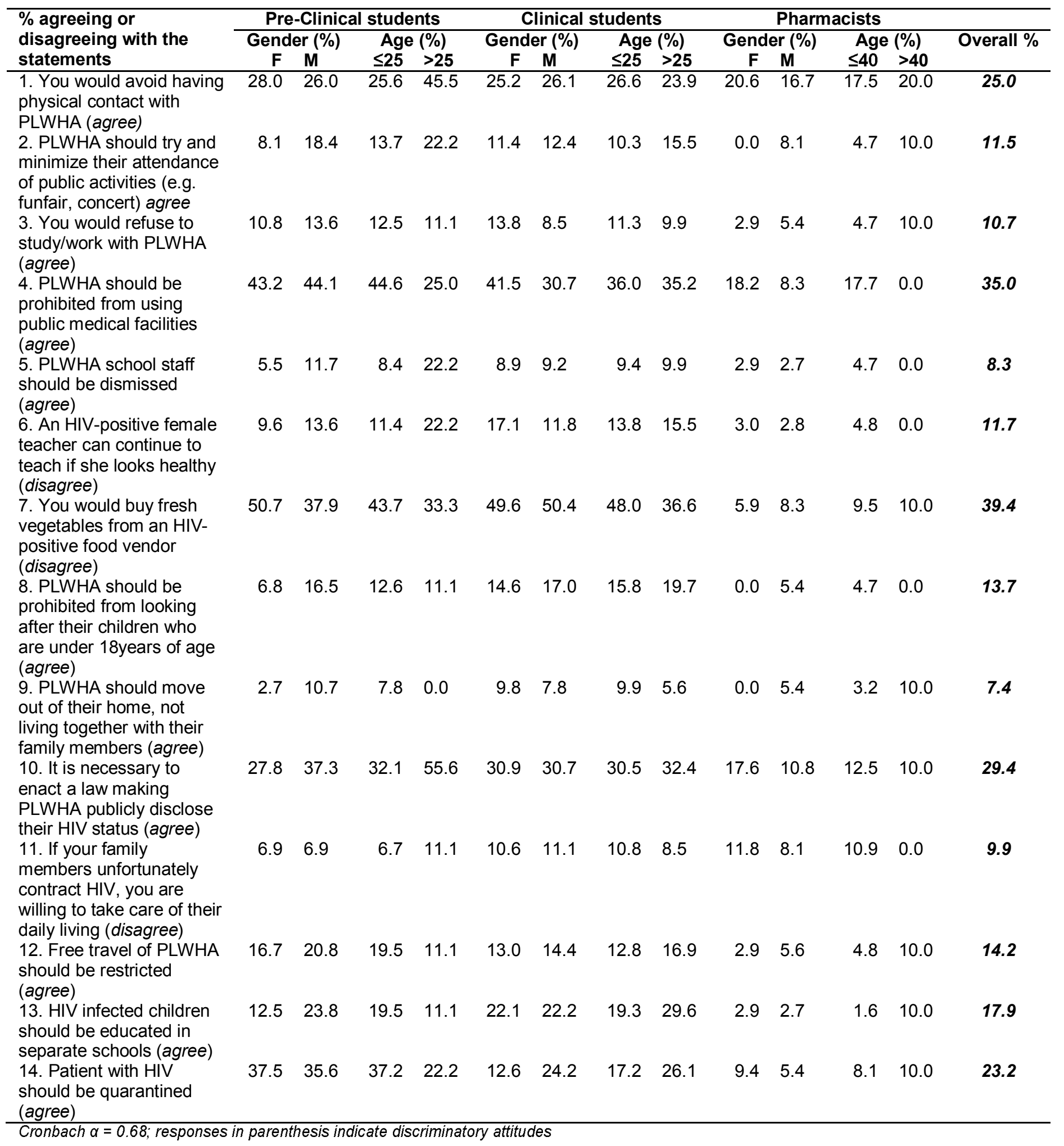

The first model showed only level of professional training (Adj. $R^{2}=0.041, \beta=-0.048, p<0.0001$ ) as a predictor of discriminatory attitude. The second model indicated level of training and the perception that "PLWHA would make their colleagues apprehensive" (Adj. $\mathrm{R}^{2}=0.066, p<$ 0.0001 ) as predictors of discriminatory attitudes among pharmacy students and pharmacists. The third model which explained significantly more variance in discriminatory attitude than the other two models comprised level of professional training and the perceptions that "PLWHA would make their colleagues apprehensive" and "PLWHA should feel ashamed of themselves" (Adj. $\left.\mathrm{R}^{2}=0.082, p<0.0001\right)$. The results of correlation and regression were not tabulated. 
Table 4: Relationship between discriminatory attitudes and HIV knowledge and perceptions among students and pharmacists

\begin{tabular}{|c|c|c|c|c|c|c|c|c|}
\hline \multirow[t]{2}{*}{$\begin{array}{l}\% \text { agreeing or disagreeing with } \\
\text { the statements (Discriminatory } \\
\text { attitudes) }\end{array}$} & \multicolumn{2}{|c|}{$\begin{array}{c}\text { No of correct HIV } \\
\text { knowledge items } \\
(\%)\end{array}$} & \multicolumn{2}{|c|}{$\begin{array}{l}\text { People who got } \\
\text { HIVIAIDS through } \\
\text { sex deserve it }(\%) \\
\end{array}$} & \multicolumn{2}{|c|}{$\begin{array}{l}\text { PLWHA should } \\
\text { feel ashamed of } \\
\text { themselves }(\%)\end{array}$} & \multicolumn{2}{|c|}{$\begin{array}{l}\text { PLWHA would } \\
\text { make colleagues } \\
\text { apprehensive (\%) }\end{array}$} \\
\hline & $\begin{array}{r}0-10 \\
\text { items }\end{array}$ & $\begin{array}{l}11-15 \\
\text { items }\end{array}$ & Agree & Disagree & Agree & Disagree & Agree & Disagree \\
\hline $\begin{array}{l}\text { 1. You would avoid having physical } \\
\text { contact with PLWHA (e.g. not } \\
\text { swimming/playing basketball with } \\
\text { them) (agree) }\end{array}$ & 25.5 & 24.3 & 32.7 & 23.2 & 28.9 & 24.7 & 28.4 & 21.4 \\
\hline $\begin{array}{l}\text { 2. PLWHA should try and minimize } \\
\text { their attendance of public activities } \\
\text { (e.g. funfair, concert) agree }\end{array}$ & 13.2 & 10.0 & 12.3 & 11.1 & 11.1 & 11.6 & 11.6 & 11.4 \\
\hline $\begin{array}{l}\text { 3. You would refuse to study/work } \\
\text { with PLWHA (agree) }\end{array}$ & 12.3 & 9.6 & 13.2 & 10.1 & 24.4 & 9.2 & 13.1 & 8.7 \\
\hline $\begin{array}{l}\text { 4. PLWHA should be prohibited from } \\
\text { using public medical facilities } \\
\text { (agree) }\end{array}$ & 40.7 & 31.5 & 40.6 & 33.5 & 42.2 & 34.8 & 39.5 & 31.4 \\
\hline $\begin{array}{l}\text { 5. PLWHA school staff should be } \\
\text { dismissed (agree) }\end{array}$ & 9.7 & 7.4 & 4.7 & 9.4 & 15.6 & 7.7 & 9.4 & 7.1 \\
\hline $\begin{array}{l}\text { 6. An HIV-positive female teacher } \\
\text { can continue to teach if she looks } \\
\text { healthy (disagree) }\end{array}$ & 13.7 & 10.3 & 11.4 & 11.8 & 15.6 & 11.6 & 13.5 & 9.1 \\
\hline $\begin{array}{l}\text { 7. You would buy fresh vegetables } \\
\text { from an HIV-positive food vendor } \\
\text { (disagree) }\end{array}$ & 47.5 & 33.2 & 53.4 & 35.8 & 60.0 & 36.9 & 44.1 & 32.8 \\
\hline $\begin{array}{l}\text { 8. PLWHA should be prohibited from } \\
\text { looking after their children who are } \\
\text { under } 18 \text { years of age (agree) }\end{array}$ & 18.6 & 9.1 & 15.1 & 13.7 & 13.3 & 13.3 & 15.4 & 13.0 \\
\hline $\begin{array}{l}\text { 9. PLWHA should move out of their } \\
\text { home, not living together with their } \\
\text { family members (agree) }\end{array}$ & 10.4 & 5.4 & 6.6 & 7.8 & 13.3 & 7.1 & 7.5 & 7.5 \\
\hline $\begin{array}{l}\text { 10. It is necessary to enact a law } \\
\text { making PLWHA publicly disclose } \\
\text { their HIV status (agree) }\end{array}$ & 31.0 & 28.3 & 35.6 & 27.6 & 31.1 & 29.0 & 32.6 & 27.2 \\
\hline $\begin{array}{l}\text { 11. If your family members } \\
\text { unfortunately contract HIV, you are } \\
\text { willing to take care of their daily } \\
\text { living (disagree) }\end{array}$ & 9.3 & 10.3 & 13.2 & 9.2 & 11.1 & 8.8 & 11.6 & 7.9 \\
\hline $\begin{array}{l}\text { 12. Free travel of PLWHA should be } \\
\text { restricted (agree) }\end{array}$ & 18.3 & 11.2 & 21.2 & 12.3 & 24.4 & 13.1 & 18.3 & 9.8 \\
\hline $\begin{array}{l}\text { 13. HIV infected children should be } \\
\text { educated in separate schools } \\
\text { (agree) }\end{array}$ & 21.6 & 16.2 & 21.7 & 17.3 & 40.0 & 16.6 & 20.3 & 15.7 \\
\hline $\begin{array}{l}\text { 14. Patient with HIV should be } \\
\text { quarantined (agree) }\end{array}$ & 28.2 & 20.0 & 29.5 & 21.6 & 45.5 & 21.4 & 27.7 & 19.2 \\
\hline
\end{tabular}

Note: Responses in parenthesis indicate discriminatory attitudes towards PLWHA

\section{DISCUSSION}

This study reports a high prevalence of discriminatory attitudes towards PLWHA among students and pharmacists in Nigeria. It also showed the effects of knowledge status and negative perceptions on the level of discriminatory attitudes directed against PLWHA.

Discriminatory attitudes towards PLWHA were common in this study especially with the student respondents. Pharmacy students declined having any contact or buy fresh vegetables from PLHWA possibly for the "fear" of being contaminated if such happened. In a similar study assessing knowledge and attitudes towards HIVIAIDS, pharmacists and pharmacy students in Zimbabwe, over-estimated their "fear" of contracting the virus even when they were well knowledgeable of its transmission [13]. Paradoxically the fear and worry of contracting the virus may eventually put such an individual at a lower risk and favor prevention $[14,15]$. Onethird $(31 \%)$ of pharmacists in this study possessed accepting attitudes in all 14 discriminatory attitude indicators, a contrast with the Ghanaian study reporting only $17 \%$ acceptance in 4 indicators [11].

Poor knowledge of HIVIAIDS is significantly associated with increased discriminatory attitudes towards PLWHA as reported in other studies [4,16]. However, when adjusted with other predictors of discriminatory attitudes, HIVIAIDS knowledge status did not predict discriminatory attitudes among students and pharmacists in our study. This pattern has been reported in another study [12]. 
To being ashamed for being HIV positive and the perceived apprehensive effect of PLWHA on other colleagues played a significant role in the stigmatization of this vulnerable group. This result confirms the assertions made in the Ghanaian study where it noted that judgmental attitudes could cause discrimination of PLWHA [11]. When pharmacists perceive their HIV patients negatively, patient-provider trust may be difficult to build during counseling and patient attrition during service delivery is almost inevitable.

Increasing professional training from pre-clinical to actual practice has been shown in this study to improve knowledge and reduce discriminatory attitudes and negative perceptions towards PLWHA. A similar study conducted using medical students of varying educational levels and residents showed that increased training diminished any discrimination towards treating PLWHA [17]. It is suggested therefore that as pharmacy students progress through their professional training, social aspects of HIVIAIDS education and strict professionalism should be inculcated into the curriculum to further improve perceptions and attitudes towards PLWHA.

Stigmatization of PLWHA in Nigeria among the general population has been reported to be high and it is quite worrying when health professionals form part of that group [18]. Pharmacists are beginning to have more contact with HIVIAIDS patients and they are usually the first port of call of illness reporting in most cases [11]. Thus their attitude towards and perceptions of PLWHA is very important for effective care and be given serious thought if we are to kick discrimination and stigmatization of PLWHA out of our health care system.

We believe future studies conducted in other universities and regions of the country will give a more robust understanding of the prevalence of stigmatization among pharmacists and if geographical location influence discriminatory patterns. Secondly, the scope of this present study did not include incidence of actual discrimination towards PLWHA by pharmacists or clinical students, and that has formed the focus of a new and ongoing research. Furthermore, there is a need to explore other possible causal factors that may have contributed to these discriminatory attitudes exhibited by pharmacists and pharmacy students.

This study suffered some limitations. Participants in this study were all drawn from students and graduates of one university and pharmacists from two university teaching hospitals, so the reader is cautioned from generalizing the study results to pharmacists and students from other universities. As with studies evaluating attitude and perceptions, responses from participants may have been biased most especially as they might want to "sound" or "look" positive to questions addressing discriminatory attitudes and negative perceptions. It is hoped that anonymity and confidentiality promised reduced such bias to the minimum.

\section{CONCLUSION}

Discriminatory attitudes against PLWHA among pharmacy students and pharmacists in this study were high. Being a student and having negative perceptions such as "PLWHA should feel ashamed of themselves" and "PLWHA would make their colleagues apprehensive was associated with these discriminatory attitudes. Increase in professional training and awareness on better perceptions might reduce discrimination against PLWHA. Comparative and longitudinal studies should be conducted to assess interventions being introduced to reduce stigmatization of PLWHA.

\section{ACKNOWLEDGEMENT}

Our appreciation goes to Vinco Educational Fund, Vinco Pharma Ltd, Nigeria which partly funded the data collection phase of this research as an undergraduate student grant. We also acknowledge the efforts of Pharmacists Austin Ezeugwu and Ogechukwu Arum-Ubaka during the data collection phase of the study.

\section{REFERENCES}

1. O'Hea EL, Sytsma SE, Copeland A, Brantley PJ. The attitude towards women with HIVIAIDS scale (ATWAS): Development and Validation. AIDS Educ Prev. 2001; 13(2): 120-130

2. Quach L, Mayer K, McGarvey ST, Lurie MN, Do P. Knowlegde, attitudes and practices among physicians on HIVIAIDS in Quang Ninh, Vietnam. AIDS Patient Care ST. 2005; 19(5): 335-346

3. Andrewin $A$, Chien L. Stigmatization of patients with HIVIAIDS among doctors and nurses in Belize. AIDS Patient Care ST. 2008; 22(11): 897-906

4. Chen W, Han M, Holzemer WL. Nurses' knowledge, attitude and practices related to HIV transmission in Northeastern China. AIDS Patient Care ST. 2004; 18(7): 417-422

5. Letamo G. The discriminatory attitudes of health workers against people living with HIV. PLoS Med. 2005; 2(8): $e 261$

6. Oyeyemi A, Oyeyemi A, Abegbune A. Knowledge, attitude and willingness of Nigerian physiotherapists to provide care for patients living with AIDS. Physio Res Inter. 2008; 13(3): 176-188

7. McQuarrie K, Echaus T, Nyblade L. HIV-related stigma and discrimination: a summary of recent literature. UNAIDS, 2009; pp 1-15. 
8. Adeneye AK, WR Brieger, Mafe MA, Adeneye AA, Salami KK, Titiloye MA, Adewole TA, Agomo PU. Willingness to seek HIV testing and counseling among pregnant women attending antenatal clinics in Ogun State, Nigeria. Inter Quart Com Health Educ. 2006; 26(4): 337-353.

9. Chisholm MA, Ricci J. Development and cross-validation of an instrument to measure first year pharmacy students' attitude toward AIDS/HIV patients. Am J Pharm Educ. 1998; 62: 162-166

10. Ahmed SI, Hassali MA, Aziz NA. An assessment of knowledge, attitudes and risk perceptions of pharmacy students regarding HIVIAIDS. Am J Pharm Educ. 2009; 73(1): Article 15.

11. Owusu-Daaku FTK, Buanya-Mensah G. Ghanaian pharmacists' perception of people living with HIV and AIDS (PLWHIV). J Pharm Pract. 2010; 23 . 135-139

12. Lau JTF, Ysui HY. Discriminatory attitudes toward people living with HIVIAIDS and associated factors: a population based study in the Chinese general population. Sex Transm Infect. 2005; 81: 113-119
13. Ball DE, Mazarurwi P. HIVIAIDS knowledge and attitudes amongst pharmacists in Zimbabwe. Cent Afr J Med. 2003; 49: 27-31

14. Rosenstock I.M., Strecher V.J., Becker M.H. The health belief model and HIV risk behavior change. In: DiClemente RJ, Peterson JL (eds), Preventing AIDS: theories and methods of behavioral interventions. Plenum Press, New York, 1994; pp 5-24.

15. Falck RS, Siegal HA, Wang J, Carlson RG. Usefulness of the health belief model in predicting HIV needle risk practices among injection drug users. AIDS Educ Prev. 1995; 7: 523-33.

16. Dadkha B, Mohammadi MA, Mozafari N. Knowledge and attitude towards HIVIAIDS among college students in Ardabil, Iran. Res J Biol Sci. 2008; 3(1): 28-31

17. Radecki S, Shapiro J, Thrupp LD, Gandhi SM, Sangha SS, Miller RB. Willingness to treat HIV-Positive Patients at different stages of medical education and experience. AIDS Patient Care ST. 1999; 13(7): 403-414

18. Babalola, S., A. Fatusi and J. Anyanti. "Media saturation, communication exposure and HIV stigma in Nigeria." Soc Sci Med. 2009; 68(8): 1513-1520. 